\section{RF16 AN AGENT-BASED MODEL OF SOCIAL CARE SUPPLY AND DEMAND IN THE UK}

ES Silverman*, U Gostoli. MRC/CSO Social and Public Health Sciences Unit, University of Glasgow, Glasgow, UK

\subsection{6/jech-2018-SSMabstracts.105}

Background Social care provision is vital for ensuring the health of ageing and vulnerable populations. The UK relies on informal care for $50 \%$ of care provision, meaning that social care policies have significant implications for health services sustainability in this context. We present an agent-based simulation of UK informal care provision, demonstrating how this framework captures troubling trends and inequalities in social care.

Methods We constructed an agent-based model in Python that simulates individual human agents in a virtual UK from the year 1860 to 2022. Population dynamics are driven by UK birth rates and mortality rates. Agents can form partnerships, reproduce, migrate domestically for work or other purposes, change jobs, and provide social care. Care decisions are taken based on employment status, salary, age, health status, geographical location, and their relationship to those in need of care. Simulated agents participate in a detailed economy, and are members of different socioeconomic status groups depending on their income.

Output files track agents' socioeconomic status, social mobility, informal care provision, and payment for formal care services. Simulation output includes individual-level agent statistics and population-level analyses of care provision by age, sex, socioeconomic status, and employment status. Simulation results were calibrated against 2011 UK Census data for key population dynamics measures.

Results Simulation results in the year 2022 show significant inequalities in social care need and provision by gender and SES group. Agents in the lowest SES quintile (Group I) show a mean unmet care need of 19 hours/week, as compared to $12.5 /$ week in in the highest (Group V). Carers in Group I supply an average 8.6 hours/week of care, compared to 3.6 hours/week in Group V. Thus, agents in Group I not only make a lower wage, they also lose more hours of work to care provision, and need more care themselves. In addition, female agents provide 1.9 times more informal care than males, while receiving lower average wages. Finally, the simulation shows a trend of growth in unmet care need from 1.17 hours per capita in 1976 to 2.38 by 2022 .

Conclusion This work demonstrates that a well-constructed agent-based simulation can provide a platform for investigating the influence of economic and social factors on social care provision. This framework thus provides a means to develop and test new social care policies which better account for the complexities and challenges facing informal carers across the country, and in turn better protect health services sustainability.

\section{RF17 WHAT MAKES ADOLESCENTS BINGE DRINK SO OFTEN? RESEARCH EVIDENCE FROM A POPULATION SCHOOL SURVEY IN CHILE}

${ }^{1} \mathrm{MF}$ Roman, ${ }^{2} \mathrm{~N}$ Cable*. ${ }^{1}$ Psychology, Universidad de La Frontera, Temuco, Chile; ${ }^{2}$ Epidemiology and Public Health, University College London, London, UK

10.1136/jech-2018-SSMabstracts. 106
Background Binge drinking is known to cause alcohol-related harm among young people. Although the link between adolescent binge drinking and adolescents' own, parental or peer factors were established, little is known about the factors that associate with the frequency of binge drinking in the school contexts. The aim of this study is to examine the contextual associations between parental, peer and school factors and the frequency of binge drinking among Chilean school children aged 13 to 18 . We hypothesised that severe school level deprivation would be associated with an increased number of binge drinking events as well as lower levels of parental supervision, and parental and peer drinking.

Data Information on frequency of binge drinking in the past month, parental supervision, paternal and maternal drinking and peer drinking was extracted from the Tenth Chilean School Population National Substance Use Survey conducted in 2013. Frequency of binge drinking was analysed among those reporting alcohol use. The individual-level information was linked to school-level information (percentage of free school meal children) obtained from the Ministry of Education

Methods Hierarchical data, individuals $(n=41,146)$ nested within schools $(n=1,687)$, were analysed using multilevel zeroinflated Poisson regression. Coefficients from the Poisson part were exponentiated to obtain Incidence Rate Ratios (IRR). Estimates were adjusted for parental education, child's age and school type and boys and girls were analysed separately.

Results Results from the Poisson part in the final model showed significant associations between lower levels of parental supervision and increased binge drinking frequencies in boys and girls. For girls, maternal drinking during weekends increased their binge drinking episodes by $10 \%$ (IRR $=1.11$ 95\% CI 1.03; 1.20), while mother's daily drinking habits increased them by $24 \% \quad($ IRR $=1.2495 \%$ CI $1.18 ; 1.38)$. Maternal daily drinking also showed increases in boy's binging episodes by $21 \%(\mathrm{IRR}=1.2195 \%$ CI $1.11 ; 1.31)$. Having at least half of friends that consumed alcohol increased the average number of binge drinking episodes by $21 \% \quad(\mathrm{IRR}=1.21$ $95 \%$ CI $1.13 ; 1.28)$ for boys, but by $60 \%$ (IRR $=1.5895 \% \mathrm{CI}$ $1.45 ; 1.71)$ for girls. School deprivation was positively associated with the number of events of binge drinking for girls only (IRR $=1.0034$ 95\% CI 1.002; 1.005).

Norms, especially mother's supporting alcohol use and peer influences were major contributor for frequent binge drinking among Chilean adolescents. Girls are likely to be more vulnerable to frequent binge drinking, influenced by their peer groups and school environment.

\section{RF18 VARIABLE SELECTION AND DATA REDUCTION FOR THE DEVELOPMENT OF A SMALL AREA DEPRIVATION INDEX FOR HEALTH RESEARCH IN BRAZIL}

${ }^{1}$ DO Ramos ${ }^{* 1,2} \mathrm{MYT}$ Ichihara, ${ }^{1,3} \mathrm{P}$ Rebouças, ${ }^{1,3} \mathrm{FJ}$ Oliveira, ${ }^{1,3} \mathrm{~A}$ Ferreira, ${ }^{3} \mathrm{C}$ Teixeira, ${ }^{2,4} \mathrm{SV}$ Katikireddi, ${ }^{1,2,3} \mathrm{ML}$ Barreto, ${ }^{2,4} \mathrm{~A}$ Leyland, ${ }^{2,4} \mathrm{R}$ Dundas. ${ }^{1}$ Center for Data Integration and Health Knowledge, Fiocruz Bahia, Salvador, Brazil; ${ }^{2}$ on behalf of the NIHR Global Health Research Group on Social Policy and Health Inequalities; ${ }^{3}$ Graduate School of Public Health, Federal University of Bahia (UFBA), Salvador, Brazil; ${ }^{4}$ Social and Public Health Sciences Unit, MRC/CSO, Glasgow, UK

\subsection{6/jech-2018-SSMabstracts. 107}

Background People living in areas with higher material deprivation have poorer health and increased mortality. In order to study these inequalities context-specific indicators of material 